\title{
Optical guidelines and signal quality for LDA applications in circular pipes
}

\author{
Zh. Zhang
}

\begin{abstract}
The optical performance of laser Doppler anemometer (LDA) technology in applications to circular pipes with an external plane wall has been clarified and quantified. It is shown that optical aberration is a persistent feature in such LDA measurements and measurements from each direction along a full pipe diameter are needed to obtain the flow distribution. For measurements of axial velocities in a circular pipe no special care has to be taken, even if the optical plane deviates from the pipe axis. For measurements of tangential and radial velocities detailed operating guidelines have been presented with respect to the shift of the measurement volume, its optical properties and the beam waist dislocations. The analysis reveals the possible influences on both the signal quality and the measurement accuracy.
\end{abstract}

\section{1}

\section{Introduction}

Fluid flow in a circular pipe is one of the most familiar and well-understood flows, but its measurement with laser Doppler anemometry (LDA) is not at all straightforward because of the surface curvatures on both the inside and the outside of the pipe. For instance simultaneous twocomponent velocity measurements using four laser beams cannot be carried out, as the four beams do not intersect at a single point in the flow owing to the optical aberration. This optical aberration is comparable to that which also occurs with off-axis alignment of the LDA unit when the optical axis intersects a plane wall at an angle other than a right angle, a phenomenon that is known as astigmatism (Zhang 1993, 1995; Zhang and Eisele 1995, 1996, 1998a).

Received: 16 May 2003 / Accepted: 19 December 2003

Published online: 31 March 2004

(C) Springer-Verlag 2004

\section{Z. Zhang $(\square)$}

SulzerInnotec, 8401 Winterthur, Switzerland

E-mail: z.zhang@fh-aargau.ch

Present address: Z. Zhang

Faculty of Science and Technology, University of Applied Sciences Aargau, 5210 Windisch, Switzerland

The present work originated during an experimental measurement campaign for an industrial project named by JET, which aims to investigate the free surface jet in Pelton turbines. The author greatly thanks PSEL (Fund for Projects and Studies of the Swiss Electric Utilities) and Sulzer Markets Technology for their financial support.
Nearly all of the optical features and the effects of astigmatism that are present in off-axis alignment of the LDA unit to a plane window also exist in a similar form in attempts to measure flows in a circular pipe.

In such circumstances, LDA applications suffer from the optical aberration related to the LDA transmitting/ receiving optics and the optical aberration related to each individual laser beam. The optical aberration related to the LDA optics implies a displacement between two measurement volumes of a two-component LDA unit, so that only one-component velocity measurements are possible. The optical aberration related to the individual laser beam leads to a deformation of the beam waist, causing a distortion of LDA measurement volume. Laser beam refraction on the curved interfaces of circular pipes requires calculations of beam transmittance with respect to both the intersection position and the intersection angle of laser beams. To avoid the difficulty of calculating the laser beam refractions on the curved flow interface, a simple method of matching the refractive index of the test fluid to that of the pipe wall has often been applied in small-scale laboratory measurements. It is indeed an effective method that also enables simultaneous two-component measurements to be made. It is, however, not applicable in most industrial applications, where the refractive index matching is impossible or gaseous flow is encountered.

In dealing with flow measurements in a circular pipe by means of the LDA method without refractive index matching, the optical performance can be considerably improved if the outside of the pipe is cut off and made plane, as illustrated in Fig. 1 from a recent industrial application (Zhang and Parkinson 2002). This approach was initially carried out to simplify the laser beam transmittance calculation, as the formation of the measurement volume becomes independent of the thickness of the pipe wall. The approach, however, additionally makes a contribution to the reduction of optical aberration, so that optical aberration is only restricted to the beam refraction on the internal surface of the circular pipe. The use of a moveable window in Fig. 1 makes the equivalent plane cut off and enables the free alignment of LDA to the pipe, so that the flow distribution in the pipe cross-section could be measured. Measurements of all three velocity components clearly still have to be carried out separately.

Simplified calculation processes generally exist for the position and the shift of the measurement volume for LDA applications in circular pipes (Boadway and Karahan 1981), but they are not valid for the current case with a plane wall at the outside of the pipe and do not consider 

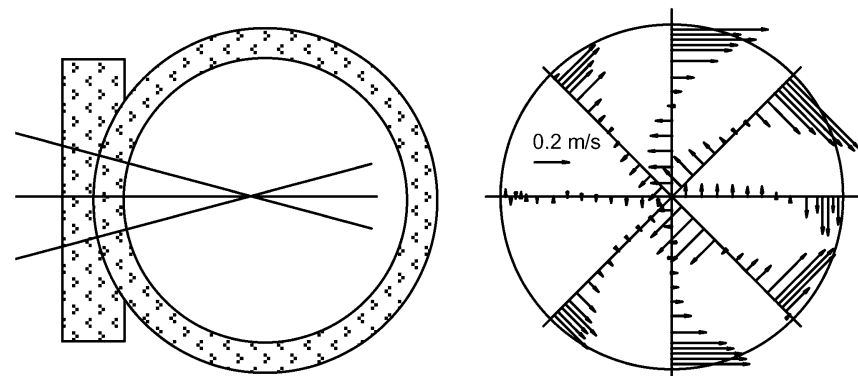

Fig. 1. Plane wall configuration on the outside of a circular pipe for LDA applications (Zhang and Parkinson 2002)

the change in optical performance due to the optical aberration. Even when using the plane walls at the outside of the circular pipe, the remaining optical aberration occurring at the internal surface still deteriorates the LDA performance. As described by Zhang and Eisele (1995, 1998a) with respect to the effects of astigmatism, the optical aberration related to the LDA optics implies that only a few elementary segments on the LDA receiving lens can see the measurement volume. This results in a decrease in the velocity signal quality and in a brisk reduction of the signal rate. Experience shows that in traversing a diameter across a circular pipe, the available signal quality and thus the signal rate could be achieved only within a depth of about a third of the pipe diameter, if no plane wall cut-off on the outside of the circular pipe is applied. As a result of using the plane wall cut-off, however, velocity signals of sufficiently high quality could be obtained even at a depth of about two-thirds of the pipe diameter. This feature of LDA measurements applies both to the axial and the tangential velocities. It is also independent of the pipe diameter because for a given relative depth of the measurement volume in the pipe, the backward scattered laser lights are mapped through the curved pipe wall to the receiving optics in the same proportion. To get the complete distribution of each velocity component across the pipe, measurements need to be performed additionally from the opposite side by rotating the LDA unit $180^{\circ}$ around the pipe axis. This two-measurement feature for a complete velocity profile represents just the optical characteristics of the circular pipe flow.

Another serious aspect associated with laser beam refraction on the curved interface, which also affects the signal qualities, is that the measurement volume and the laser beam waists are no longer coincident. For large differences in position between the measurement volume and the waists of the beams, the laser light intensity in the measurement volume becomes very low, so that the velocity signals will become too weak to be detected. Furthermore, the dislocation of laser beam waists inevitably leads to the fringe distortion in the measurement volume, so that the measurement accuracy will be affected.

In the present paper, methods of calculating the measurement volumes, both for axial, tangential and radial velocities, will be shown for LDA applications to the circular pipe with a plane wall cut-off on the outside wall of the pipe. The beam waist dislocations will be accounted for to give a reference for possible measurement errors especially for tangential and radial velocities. In all cases, the optical axis of LDA is aligned perpendicular to the plane of the cut-off. This ensures that the optical aberration associated with the laser beam transmittance through the cutoff plane is negligible. In considering the laser beam refraction on the circular interface (wall-fluid), the local surface curvature will be neglected. This is validated because the laser beam diameter, in reality, is negligibly small against the pipe radius.

\section{2}

\section{Measurements of axial velocities}

Measurements of axial velocities require that the two laser beams lie in a plane parallel to the pipe axis. By arranging the plane containing the two laser beams (designated as the optical plane) to go through the pipe axis, the laser beam refraction on the internal wall of the circular pipe is comparable to that on a perpendicular plane surface. The optical aberration then tends to be kept to a minimum, so that the best available optical condition is obtained.

A particular property of the optical layout for measurements of axial velocities is highlighted here for future reference. As long as the optical plane is kept parallel to the pipe axis, without having to go through it, the intersection angle between the two refracted laser beams remains unchanged. According to Fig. 2 and with regard to the refraction law, the $z$ component $a_{2 z}$ of the unit vector $\vec{a}_{2}$ which denotes the refracted laser beam $\mathrm{A}$ is equal to $a_{2 z}=n_{1} / n_{2} \cdot a_{1 z}$ (only true, if the surface normal $\vec{n}$ is perpendicular to the $z$ coordinate). The intersection angle $2 \alpha_{2}$ between the two refracted laser beams A and B then can be represented by:

$\cos 2 \alpha_{2}=\vec{a}_{2} \cdot \vec{b}_{2}$

Owing to the symmetry condition $\left(a_{2 x}=b_{2 x}, a_{2 y}=b_{2 y}\right.$ and $\left.a_{2 z}=-b_{2 z}\right)$ and $a_{2 x}^{2}+a_{2 y}^{2}=1-a_{2 z}^{2}$ for the unit vector $\vec{a}_{2}$ it results from Eq. 1

$\cos 2 \alpha_{2}=1-2 a_{2 z}^{2}=1-2\left(n_{1} / n_{2} \cdot a_{1 z}\right)^{2}$

or with the half intersection angle and $a_{1 z}=\sin \alpha_{1}$ for the laser beam A prior to the refraction:

$\sin \alpha_{2}=n_{1} / n_{2} \cdot a_{1 z}=n_{1} / n_{2} \cdot \sin \alpha_{1}$

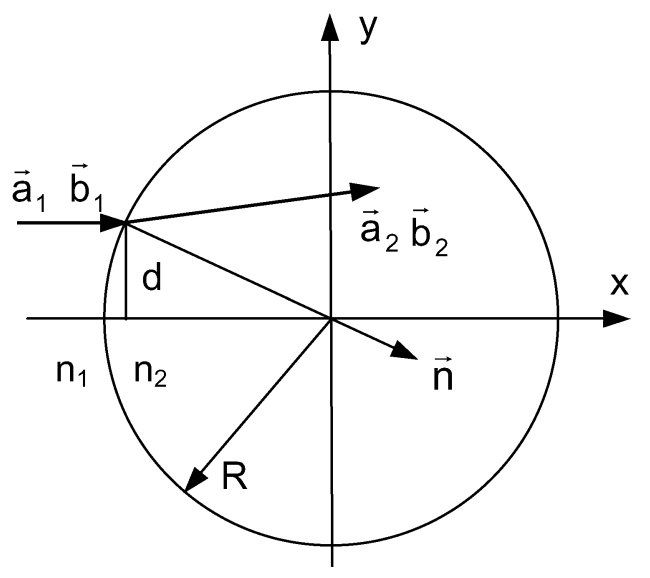

Fig. 2. Deviation of the optical plane from the pipe axis in measuring axial velocities 
As can be seen the intersection angle of the two laser beams and thus the optical properties of the measurement volume in the test fluid remain unchanged, in spite of the deviation of the optical plane from the pipe axis. This result indicates that the deviation in aligning the optical plane from going through the pipe axis does not lead to any error in velocity measurements. The analysis made here can be easily demonstrated by a table top experiment when a scaled rule is inserted into a circular pipe with or without water. The observation from the outside shows that there is no optical distortion occurs with the rule scale in the axial direction. The distortion occurs only in the radial direction.

Although the deviation of the optical plane from the pipe axis does not lead to any change in the beam intersection angle, there are, however, other aspects that should be accounted for:

- With the displacement of the LDA unit along the $x$ direction the measurement volume in the test fluid is moved along the bisector of two refracted laser beams, i.e. along a path that is not parallel to the $x$ axis. Note that the bisector of the two refracted laser beams does not precisely coincide with the refracted optical axis. This phenomenon is known as comatic aberration. In the present case the difference between them can be neglected to a first approximation.

- In regarding the shift of the measurement volume along the bisector of two refracted laser beams, the ratio between this shift and the displacement of LDA unit along the $x$ axis is precisely the same, as no deviation of the optical plane from the pipe axis occurs. The $x$ component of this measurement volume shift therefore depends on the deviation considered here. It can, however, be verified that even at a deviation of $d / R=0.5$ the inclination angle of the refracted optical axis is less than $5^{\circ}$ (see Sect. 4and Fig. 8). The $x$ component of the shift of the measurement volume thus is only about $0.4 \%$ smaller than that along the refracted optical axis.

- With large deviations of the optical plane from the pipe axis the angle between the optical axis and the normal $\vec{n}$ of the interface then becomes large. This is comparable with the off-axis alignment of the LDA unit at the large off-axis angles to a plane interface, as treated by Zhang and Eisele (1995). As a result the optical aberration related to the LDA optics, or accurately speaking, the effect of astigmatism becomes significant and the quality of velocity signals will be considerably deteriorated. This optical aberration can also be visualized with the table top experiment made above, at which the scale on the rule in the pipe becomes continuously more unclear along the pipe radius.

Since the deviation of the LDA optical plane from the pipe axis brings about undesirable features, large deviations should be avoided. For moderate deviations (say $d / R<0.5$ ), as long as the signal rate is sufficiently high, no particular attention needs to be paid to this. The fringe distortion in the measurement volume, which results from the dislocation of laser beam waists and which is dependent on the deviation of LDA optical plane from the pipe axis, is anyhow not evident and will not be treated.

\section{3}

\section{Measurements of tangential velocities}

For measurements of tangential velocities the laser beams are aligned, so that the optical plane is perpendicular to the pipe axis. In regarding the laser beam refractions on the circular surface, two main aspects need to be considered:

1. The shift of the measurement volume in the flow is no longer proportional to the shift of the LDA unit.

2. The intersection angle between the two refracted laser beams and thus the property of the measurement volume depend on the local position of the measurement volume in the flow.

Detailed laser beam calculations should therefore be conducted to ensure correct LDA measurements.

\section{1}

\section{Basic analysis}

For the analysis of this situation, the laser beams are again considered as they are present in the transparent pipe wall (index 1). Owing to the symmetry between two laser beams, only one beam will be considered here. The measurement volume is then formed on the symmetrical axis ( $x$ axis in Fig. 3 ) where the laser beam arrives. The laser beam is assumed to have its start position 1 at which the measurement volume is positioned at the internal side of the pipe. The shift of the laser beam to the position 2 is given by a distance $s_{1 x}$ that is about $n_{1}$ times of the movement of LDA unit in the air. The measurement volume is moved to the position $m$, while the virtual measurement volume is found at $m^{\prime}$. The application of the sine law to the triangles $o \mathrm{~cm}^{\prime}$ and $\mathrm{ocm}$, respectively, yields

$$
\begin{aligned}
& \frac{R-s_{1 x}}{\sin \left(\alpha_{1}-\varphi\right)}=\frac{R}{\sin \alpha_{1}} \\
& \frac{R-s_{2 x}}{\sin \left(\alpha_{2}-\varphi\right)}=\frac{R}{\sin \alpha_{2}}
\end{aligned}
$$

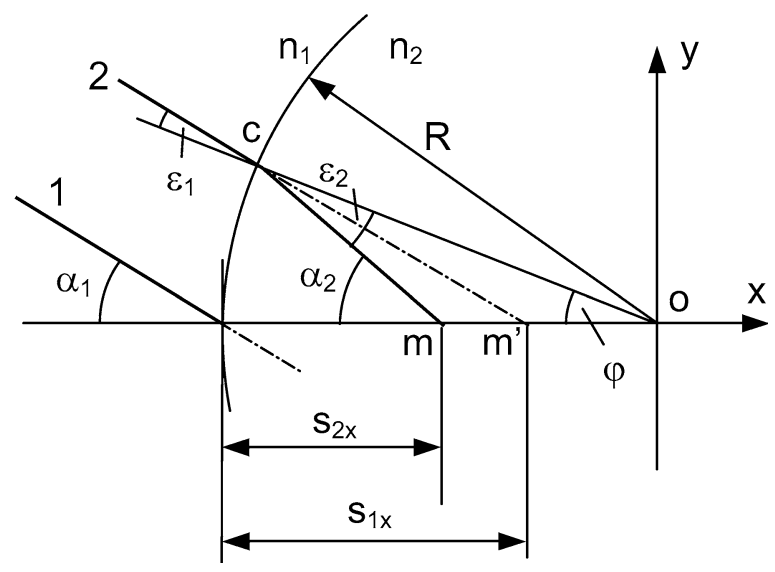

Fig. 3. Formation of the measurement volume in the flow for measurements of tangential velocities 
The refraction law in such a case is given by:

$n_{1} \sin \left(\alpha_{1}-\varphi\right)=n_{2} \sin \left(\alpha_{2}-\varphi\right)$

These three equations form the basic solutions for both the position $s_{2 x}$ and the properties $2 \alpha_{2}$ of the measurement volume in the flow. For a given movement $s_{1 x}$ the following calculating process can be performed:

$s_{1 x} \stackrel{(4)}{\rightarrow} \varphi \stackrel{(6)}{\rightarrow} \alpha_{2} \stackrel{(5)}{\rightarrow} s_{2 x}$

An application example of the presented technique can be found by Zhang and Parkinson (2002), at which the inlet flow to a model injector of a Pelton turbine was measured by the LDA method (see also Fig. 1).

\section{2}

\section{Simplifications}

In the above consideration, Eq. 7 represents the way of indirectly calculating the relevant parameters $a_{2}$ and $s_{2 x}$ to the measurement volume. This process seems to be somewhat inconvenient. With respect to the configuration of common LDA systems, at which the half intersection angle of laser beams is usually not more than $10^{\circ}$, the half intersection angle of laser beams in the transparent pipe wall is then not more than $7^{\circ}$ and the angle $\varphi$ seldom exceeds $14^{\circ}$. For this reason all the angles under the symbol of sine functions $\left(\alpha_{1}, \alpha_{2}, \alpha_{1}-\varphi\right.$ and $\left.\alpha_{2}-\varphi\right)$ in the basic equations 4,5 and 6 can be considered to be small angles. All three equations then can be simplified by using the approximation $\sin x=x$ for paraxial rays. From this paraxial approximation the characteristic parameters for the measurement volume can be directly interpreted as the function of the virtual position of the measurement volume as follows:

$\frac{s_{2 x}}{R}=\frac{1}{1+\frac{n_{1}}{n_{2}}\left(\frac{R}{s_{1 x}}-1\right)}$

$\frac{\alpha_{2}}{\alpha_{1}}=\frac{n_{1}}{n_{2}}-\left(\frac{n_{1}}{n_{2}}-1\right) \frac{s_{1 x}}{R}$

$\frac{\varphi}{\alpha_{1}}=\frac{s_{1 x}}{R}$

whereby Eq. 10 is only written for completeness. It will not be applied to quantify the measurement volume.

The inaccuracy arising from the approximation made above should be estimated. An LDA system is assumed to have a half intersection angle $\alpha_{1}=4.45^{\circ}$ between laser beams in the pipe wall $\left(n_{1}=1.52\right)$. It is also assumed that the measurement volume travels along the whole diameter of the pipe. For water flows $\left(n_{2}=1.33\right)$ the relative deviations of the quantities $s_{2 x}$ and $a_{2}$ in Eqs. 8 and 9, respectively, from those calculated from Eq. 7 are shown in Fig. 4. As can be seen the maximal error arising from the approximations leading to Eqs. 8, 9 and 10 is below $0.1 \%$ and really negligible. The same calculation has also been made for air flow $\left(n_{2}=1\right)$. The maximal error embedded in Eq. 8 is $0.4 \%$, while in Eq. 9 it is only $0.1 \%$.

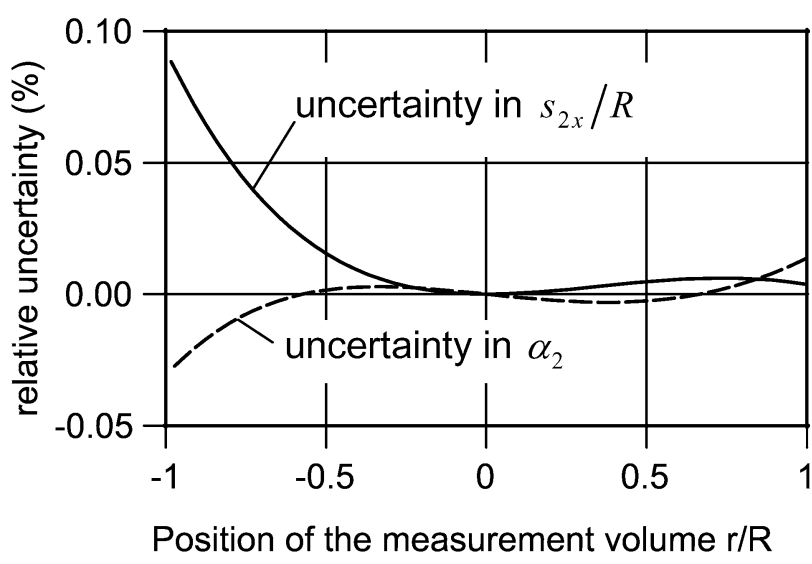

Fig. 4. Relative uncertainties in calculating the measurement volume in the water flow by using the simplified relations. The half intersection angle between the two laser beams in the pipe wall is $\alpha_{1}=4.45^{\circ}$

\section{3}

\section{Fringe spacing and velocity corrections}

In using the laser beam intersection angle in the fluid, the fringe spacing in the measurement volume is calculated as

$\Delta x=\frac{\lambda_{2}}{2 \sin \alpha_{2}}$

Because $\lambda_{1} / \lambda_{2}=n_{2} / n_{1}$ this equation is rewritten as

$\Delta x=\frac{n_{1}}{n_{2}} \frac{\sin \alpha_{1}}{\sin \alpha_{2}} \frac{\lambda_{1}}{2 \sin \alpha_{1}}=\frac{n_{1}}{n_{2}} \frac{\sin \alpha_{1}}{\sin \alpha_{2}} \Delta x_{0}$

whereby $\Delta x_{0}$ represents the initial fringe spacing which is the same for laser beams both in the air and in the pipe wall.

Equation 12 points out that the measured tangential velocity has to be corrected by a factor equal to

$k=\frac{n_{1}}{n_{2}} \frac{\sin \alpha_{1}}{\sin \alpha_{2}}$

or with respect of Eqs. 4, 5 and 6 to

$k=\frac{R-s_{2 x}}{R-s_{1 x}}$

In applying Eq. 8 this is again reformed to

$k=1+\left(n_{1} / n_{2}-1\right) \frac{s_{2 x}}{R}$

It is linearly dependent on the depth of the measurement volume.

\section{4}

\section{Measurements of radial velocities}

Measurements of radial velocities in circular pipes represent a highly complex process, if no index matching method is applied. In positioning the LDA head for radial velocity measurements according to Fig. 5, the following problems have to be solved:

- how can the measurement volume $m$ be accurately positioned?

- how can the beam intersection angle $2 \alpha_{2}$ be calculated? 


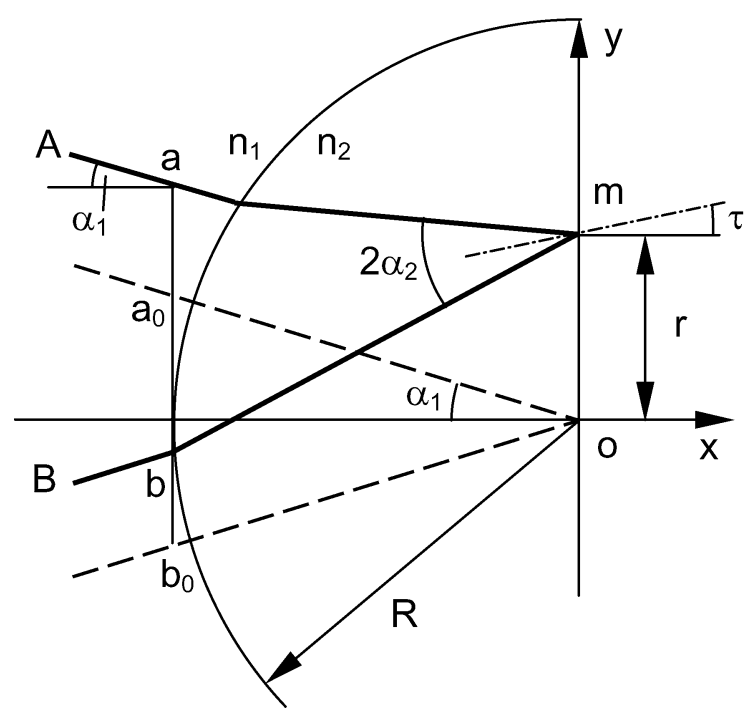

Fig. 5. Off-axis alignment of laser beams for measurements of radial velocities along the $y$-axis

- what can be said about the orientation $\tau$ of the measurement volume?

The beam intersection angle and the measurement volume orientation must be known in order to correct systematic measurement errors. In reality, because $\tau \neq 0$ the measured velocities do not represent the radial velocities.

In principle, to each LDA head position the measurement volume position in the flow has to be calculated. With the shift of the LDA unit parallel to the $y$ axis, the measurement volume would travel along a two-dimensional path. This will complicate the profile measurements of radial velocities considerably. A relatively easy method is to calculate the necessary movement of the LDA head for the given path of the measurement volume along the $y$ axis, as shown in Fig. 5. The starting point is the LDA unit position with the measurement volume at the pipe centre (basic position). This can be achieved from the method described in Sect. 3. According to Fig. 5 the necessary movement of the LDA head from the basic position to the position with the measurement volume $m$ on the $y$ axis at $r$ can be determined by:

$\Delta x_{r}=\frac{\left(y_{a}-y_{b}\right)-\left(y_{a 0}-y_{b 0}\right)}{2 \tan \alpha_{1}}$

$\Delta y_{r}=\frac{1}{2}\left(y_{a}+y_{b}\right)$

Thereby $a_{0}, b_{0}, a$ and $b$ are intersections between laser beams and the tangent of the circular pipe at $x=-R$. The basic intersection coordinates $y_{a 0}$ and $y_{b 0}$ can be simply obtained from Fig. 5 as

$y_{a 0}=R \cdot \tan \alpha_{1}$

and

$y_{b 0}=-R \cdot \tan \alpha_{1}$

Clearly detailed calculations of $y_{a}$ and $y_{b}$ for a given measurement volume position have to be performed.
4.1

\section{Calculations of intersection points $y_{a}$ and $y_{b}$}

The purpose of these calculations is to establish the relationships $y_{a}=f(r)$ and $y_{b}=f(r)$ for the given measurement volume positions at $r$. Because it deals with same calculations both for $y_{a}$ and $y_{b}$, only calculations of $y_{a}=f(r)$ for laser beam A will be shown in the following.

According to Fig. 6 the relationship $y_{a}=f(r)$ can be obtained if the relationship $\varphi_{a}=f(r)$ is known. For this reason the relationship $\varphi_{a}=f(r)$ or equivalently $r=f\left(\varphi_{a}\right)$ are first established. The position of the measurement volume on the $y$ axis is expressed by

$r=R \sin \varphi_{a}-R \cos \varphi_{a} \tan \alpha_{2 a}$

or related to $R$ :

$\frac{r}{R}=\sin \varphi_{a}-\cos \varphi_{a} \tan \alpha_{2 a}$

Here $\tan \alpha_{2 a}$ needs to be shown as a function of $\varphi_{a}$. With $\alpha_{2 a}=\varphi_{a}-\varepsilon_{2}$ and the refraction law $n_{2} \sin \varepsilon_{2}=n_{1} \sin \varepsilon_{1}$ the following relationship can be obtained:

$\tan \alpha_{2 a}=\frac{\sqrt{T_{a}-1} \tan \varphi_{a}-1}{\sqrt{T_{a}-1}+\tan \varphi_{a}}$

Thereby $T_{a}$ is given by

$T_{a}=\left(\frac{n_{2}}{n_{1}}\right)^{2} \frac{1}{\sin ^{2} \varepsilon_{1}}=\left(\frac{n_{2}}{n_{1}}\right)^{2} \frac{1}{\sin ^{2}\left(\varphi_{a}-\alpha_{1 a}\right)}$

According to Eq. 22 there has to be $T_{a} \geq 1$. In fact, $T_{a}=1$ represents just the beginning of the total reflection, as given in Eq. 23.

Together with Eq. 21 the position of the measurement volume on the $y$ axis is now shown as a function of the angle $\varphi_{a}$ :

$\frac{r}{R}=\frac{1}{\sqrt{T_{a}-1}+\tan \varphi_{a}} \frac{1}{\cos \varphi_{a}}=f\left(\varphi_{a}\right)$

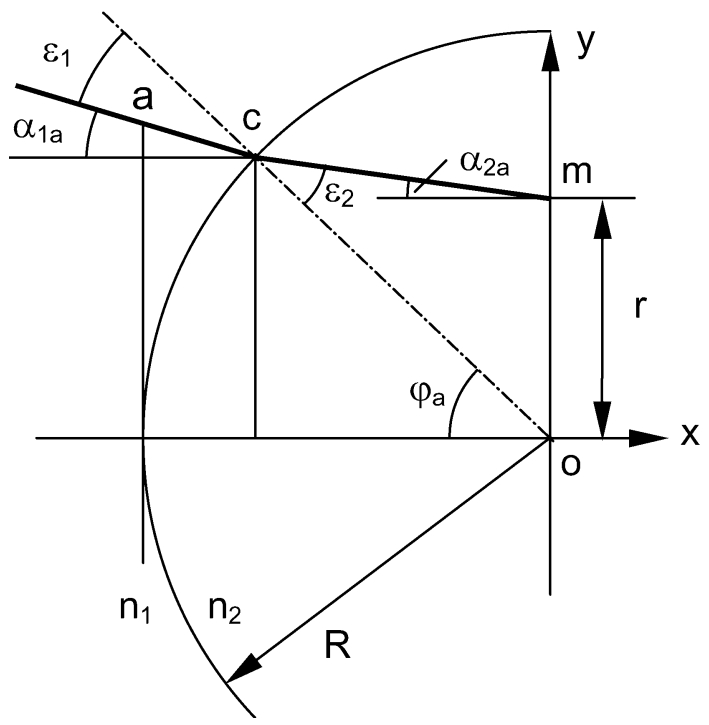

Fig. 6. Calculation of laser beam transmittance for the measurement volume on the $y$-axis 
According to Figs. 5 and 6 the angle $\varphi_{a}$ begins at $\varphi_{a}=\alpha_{1 a}$, at which the measurement volume lies in the pipe centre.

In a similar way, the same function for the laser beam $B$ can be found. The subscript $a$ in Eq. 24 needs only to be replaced by the subscript $b$. However, attention should be paid to the fact that both the angle $\alpha_{1 b}$ and $\alpha_{2 b}$ are negative for the laser beam B. Correspondingly the angle $\varphi_{b}$ begins at $\varphi_{b}=\alpha_{1 b}$.

The intersection between laser beam $\mathrm{A}$ and the tangent at $x=-R$ is calculated according to Fig. 6 as

$y_{a}=R \cdot \sin \varphi_{a}+R\left(1-\cos \varphi_{a}\right) \tan \alpha_{1 a}=f(r)$

Similarly for laser beam $\mathrm{B}$

$y_{b}=R \cdot \sin \varphi_{b}+R\left(1-\cos \varphi_{b}\right) \tan \alpha_{1 b}=f(r)$

They are both functions of $r$ because of Eq. 24 and the corresponding equation for the laser beam $B$. Together with Eqs. 16 and 17 the necessary movements of the LDA unit for a given measurement volume at $r$ can be determined.

\section{2}

\section{Simplifications}

The above two relationships given in Eqs. 25 and 26 are not explicit, because according to Eq. 24 the angle $\varphi_{a}$ can not be expressed to be an explicit function of $r / R$. For given angles $\varphi_{a}$, however, both $y_{a} / R$ and $r / R$ can be calculated, as these have been shown in Fig. 7 for a specific case. In the figure, the corresponding relationship for the laser beam $B$ is also shown. It can be concluded that below $r / R=0.8$ there exists linear relationships for both $y_{a}=f(r)$ and $y_{b}=f(r)$. By using gradients at $r / R=0$ as calculated in

$\frac{d y_{a}}{d r}=\frac{d y_{b}}{d r}=\frac{n_{2}}{n_{1}}$

intersections shown above can then be expressed as

$\frac{y_{a}}{R}=\tan \alpha_{1 a}+\frac{n_{2}}{n_{1}} \frac{r}{R}$

$\frac{y_{b}}{R}=\tan \alpha_{1 b}+\frac{n_{2}}{n_{1}} \frac{r}{R}$

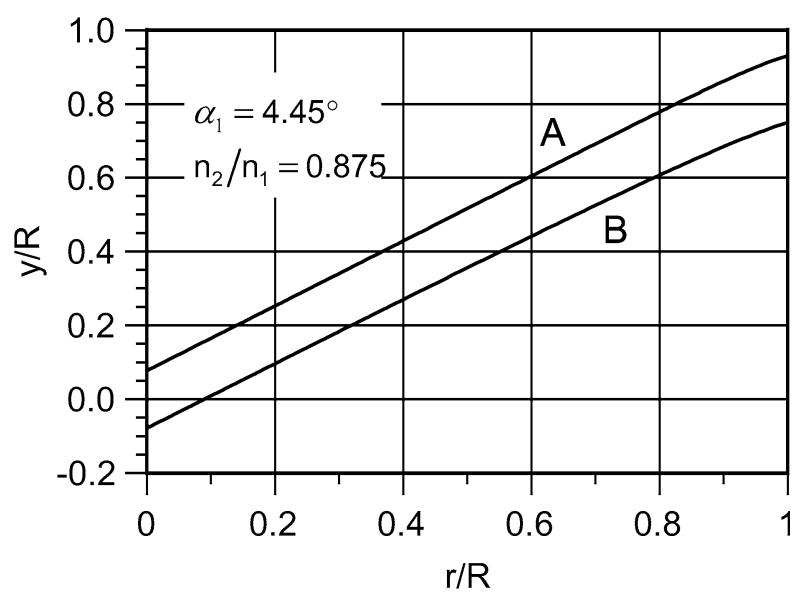

Fig. 7. Coordinates of intersections between the laser beams and the tangent at $x=-R$
Further results can be obtained from the above simplifications. Combining Eqs. 28 and 29, respectively, with Eqs. 25 and 26 yields

$\sin \left(\varphi_{a}-\alpha_{1 a}\right)=\cos \alpha_{1 a} \frac{n_{2}}{n_{1}} \frac{r}{R}$
$\sin \left(\varphi_{b}-\alpha_{1 b}\right)=\cos \alpha_{1 b} \frac{n_{2}}{n_{1}} \frac{r}{R}$

Because $\alpha_{1 a}=-\alpha_{1 b}=\alpha_{1}$ then the RHS of these two equations are equal to each other, so that

$\varphi_{a}-\varphi_{b}=\alpha_{1 a}-\alpha_{1 b}=2 \alpha_{1}$

Combining Eqs. 30 and 31, respectively, with refraction law Eq. 6 again yields

$\sin \left(\varphi_{a}-\alpha_{2 a}\right)=\cos \alpha_{1 a} \frac{r}{R}$
$\sin \left(\varphi_{b}-\alpha_{2 b}\right)=\cos \alpha_{1 b} \frac{r}{R}$

These two equations straightforwardly indicate

$\varphi_{a}-\varphi_{b}=\alpha_{2 a}-\alpha_{2 b}$

Because of Eq. 32 there is finally

$\alpha_{2 a}-\alpha_{2 b}=2 \alpha_{1}$

\section{3}

\section{LDA unit movement $\Delta x_{r}$ and $\Delta y_{r}$}

The intersection coordinates shown in Eqs. 28 and 29 are now used to calculate the necessary movements of the LDA head. Equations 16 and 17 then become

$\frac{\Delta x_{r}}{R}=0$

$\frac{\Delta y_{r}}{R}=\frac{n_{2}}{n_{1}} \frac{r}{R}$

These results point out that for measurements along the $y$ axis the LDA head needs only to be moved in the parallel direction. The ratio between the LDA movement and the shift of the measurement volume is equal to the ratio of two refractive indices.

\section{4}

Laser beam intersection angle $2 \alpha_{2}$

The intersection angle between the laser beams, which is used to correct the measurement results involving systematic errors, is calculated according to Figs. 5 and 6 as follows:

$2 \alpha_{2}=\alpha_{2 a}-\alpha_{2 b}$

Because of Eq. 36 there is

$2 \alpha_{2}=2 \alpha_{1}$

This result indicates that the beam intersection angle remains constant and is the same as that in the pipe wall. 


\section{5}

\section{Fringe spacing and velocity corrections}

In using the laser beam intersection angle in the fluid, the fringe spacing in the measurement volume is calculated to:

$\Delta x=\frac{\lambda_{2}}{2 \sin \alpha_{2}}$

Because $\alpha_{2}=\alpha_{1}$ and $\lambda_{1} / \lambda_{2}=n_{2} / n_{1}$ this equation is rewritten as

$\Delta x=\frac{n_{1}}{n_{2}} \frac{\lambda_{1}}{2 \sin \alpha_{1}}=\frac{n_{1}}{n_{2}} \Delta x_{0}$

whereby $\Delta x_{0}$ represents the initial fringe spacing.

Equation 42 points out that the measured velocities have to be corrected by a factor equal to $n_{1} / n_{2}$.

\section{6}

\section{Orientation of the measurement volume $\tau$}

The orientation of the measurement volume, i.e. the inclination of the bisector of two refracted laser beams determines the measured velocity component which generally differs from the radial velocity. The inclination angle $\tau$ as shown in Fig. 5 is considered positive and given by

$\tau=\alpha_{2}-\alpha_{2 a}=\alpha_{1}-\alpha_{2 a}$

In order to express this inclination angle as a function of $r$, Eqs. 30 and 33 are taken into account respectively so that

$$
\begin{aligned}
\tau & =\left(\varphi_{a}-\alpha_{2 a}\right)-\left(\varphi_{a}-\alpha_{1 a}\right) \\
& =\arcsin \left(\cos \alpha_{1 a} \frac{r}{R}\right)-\arcsin \left(\cos \alpha_{1 a} \frac{n_{2}}{n_{1}} \frac{r}{R}\right)
\end{aligned}
$$

Clearly this angle depends on both the optical layout $\left(\alpha_{1}\right)$ and the medium properties $\left(n_{2} / n_{1}\right)$. Figure 8 shows the inclination angle is dependent on the measurement volume position $r / R$ for a specific case with $\alpha_{1}=4.45^{\circ}$ and $n_{2} / n_{1}=0.875$. For the measurement volume within $r / R=0.8$ the inclination angle slowly increases up to $10^{\circ}$.

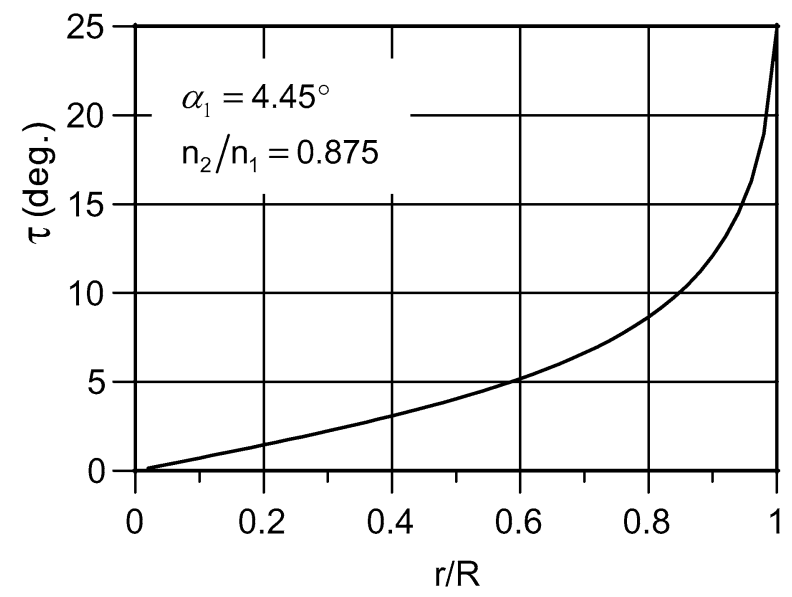

Fig. 8. Inclination angle of the bisector of the two refracted laser beams in the fluid
The inclination of the LDA optical axis after the refraction is considered here to compare with the inclination of the bisector of the two refracted laser beams, given in Eq. 44. In Fig. 6, the laser beam is replaced by the optical axis that is parallel to the $x$ axis, so that $\alpha_{1 a}=0$ and $\varepsilon_{1}=\varphi_{o}$ are obtained. The inclination angle of the refracted optical axis then is given by

$\tau_{o}=\varepsilon_{2}-\varphi_{o}$

The angle $\varphi_{o}$ is obtained from Eq. 38, which in effect represents the shift of the optical axis, so that

$\sin \varphi_{o}=\frac{n_{2}}{n_{1}} \frac{r}{R}$

In using the refraction law to represent the refraction angle $\varepsilon_{2}$ and because $\varepsilon_{1}=\varphi_{o}$, Eq. 45 then becomes

$\tau_{o}=\arcsin \left(\frac{r}{R}\right)-\arcsin \left(\frac{n_{2}}{n_{1}} \frac{r}{R}\right)$

This equation represents an approximation of Eq. 44 by assuming $\cos \alpha_{1} \approx 1$ for the small laser beam intersection angle. The difference between Eqs. 44 and 47is less than $0.4 \%$ for the case considered in Fig. 8 within the area $r / R<0.5$.

\section{7}

\section{Radial velocities $u_{r}$}

Because of $\tau \neq 0$ the measured velocity $u_{\tau}$ does not directly represent the radial velocity. In reality, the radial velocity can be obtained if the tangential velocity along the $y$ axis is accounted for. By assuming that the positive tangential velocity agrees to the $x$ direction, the radial velocity can be resolved from

$u_{\tau}=u_{r} \cos \tau-u_{t} \sin \tau$

\section{8}

\section{Remarks on the method}

The entire treatments shown above are based on the linearization according to Eqs. 28 and 29 for $r / R<0.8$. This is in any case available because measurements beyond $r /$ $R=0.5$ rapidly become impossible owing to the astigmatism and the growth of the beam section, so that the laser light intensity decreases (total reflection occurs at $T=1$ ). The calculation has shown that within $r / R=0$ to $r / R=0.5$ the maximal error in $u_{\tau}$ arising from the linearization is less than $1 \%$ (at $r / R=0.5$ ). In most engineering flows this error is quite acceptable. The error in the inclination angle $\tau$, if the Eq. 44 is considered, is less than $0.1 \%$.

The optical aberration is related to the offset of the LDA unit from the pipe centre by $r / R$ and can be represented, according to Eq. 46, by the off-axis angle $\varphi_{o}$ of the optical axis. At large values of $r / R$ with the measurement volume the off-axis angle is large. The entire optical properties then are comparable to those related to the off-axis alignment of the LDA unit to a plane wall (Zhang and Eisele 1995). For instance, for $n_{2} / n_{1}=0.875$ and $r / R=0.6$ there is $\varphi_{o}=32^{\circ}$. This off-axis angle is associated with huge astigmatism effects, so that the effective aperture of the 
receiving lens and consequently the signal rate are radically reduced (Zhang and Eisele 1998a). Although the use of a separate receiving unit positioned sideways at $90^{\circ}$ could enhance the optical aperture, the transmitting and the receiving optics, however, must always be realigned if the measurement volume is shifted to another position $r / R$. Another serious problem at the large off-axis angle is the increased probability that owing to a small inaccuracy in the optical layout both laser beams after refractions do not intersect at all (Zhang and Eisele 1998a). For this reason, it is concluded that for the measurement volume beyond $r / R=0.5$ measurements become impossible. This optical behaviour, in fact, also applies to the case for measurements of axial components with a deviation limit at $d / R<0.5$, as already discussed in Sect. 2 .

Because of Eqs. 30 and 31 it is evident from Eq. 23 that there is simply

$T_{a}=T_{b}=\frac{1}{\left(r / R \cdot \cos \alpha_{1}\right)^{2}}$

This means that under the simplification condition according to Eqs. 28 and 29 the total reflections at both laser beams would occur simultaneously.

\section{5}

\section{Optical aberration and fringe distortion}

Generally the measurement condition continuously deteriorates as the depth of the measurement volume in the test fluid increases, for instance in profile measurements of axial or tangential velocities. From experience, as mentioned in the introduction, the flow measurements can be achieved at most up to a depth of about two-thirds of the pipe diameter. The worsening of the optical condition at the upper depth is related to the optical aberration and the dislocation of laser beam waists. In the measurements of radial velocities, the worsening of signal qualities become more and more significant with the shift of the measurement volume away from the pipe centre. The optical aberration and the beam waist dislocation will be quantified in the following sections. In particular, the analysis should reveal why measurements of axial and tangential velocities in the flow area of depths beyond two-thirds of the pipe diameter become impossible. The optical aberrations that occur with the LDA arrangement for radial velocity measurements were discussed at the end of Sect. 4.

\section{1}

\section{Optical aberration}

Through the perpendicular alignment of the LDA unit to the cut-off plane on the outside of the circular pipe, any types of optical aberration arising from the beam refraction at this first media interface are negligible. To represent the optical aberration originating at the internal surface of the circular pipe the displacement between two measurement volumes for axial and tangential velocities, respectively, should be considered by assuming the use of a two-component LDA unit. This approach is equivalent to the use of the astigmatic difference to describe astigmatism. The displacement between the two measurement volumes is easily obtained from the results already obtained in Sects. 2 and 3 . The measurement volume for the axial velocity lies on the $x$ axis at a distance $\frac{n_{2}}{n_{1}} \frac{s_{1 x}}{R}$ from the pipe wall. In using the simplified Eq. 8, the displacement between the two measurement volumes is obtained by:

$\Delta s_{R}=\frac{s_{2 x}}{R}-\frac{n_{2}}{n_{1}} \frac{s_{1 x}}{R}=\frac{s_{2 x}}{R}-\frac{1}{\left(R / s_{2 x}-1\right)+n_{1} / n_{2}}$

Figure 9 shows, for given positions of the tangential measurement volume, the displacements between two measurement volumes. As can be seen, both measurement volumes separate from each other, as the depth of the measurement volume in the flow increases. In other words, the optical aberration related to the LDA optics increases with the depth of the measurement volume in the flow. Similar to effect of astigmatism that was treated for plane wall cases by Zhang and Eisele $(1995,1998 \mathrm{a})$ this optical aberration implies that the larger the depth of the measurement volume in the flow, the fewer are the effective elementary segments on the receiving lens. This immediately results in a deterioration of the velocity signals and thus in the decrease of the signal rate. For this reason it is hardly possible to obtain velocity signals of sufficiently high quality in the flow area of depths beyond two-thirds of the pipe diameter. As mentioned in the introduction, an entire flow distribution on a pipe diameter could only be achieved if an additional measurement is completed from the opposite side by rotating the LDA system $180^{\circ}$ around the pipe axis. This two-measurement feature applies to measurements of both the axial and tangential velocities. It provides, however, an opportunity for the so-called dualmeasurement method with which the tangential velocities can be exactly resolved from measurements (Zhang and Parkinson 2002).

\section{2 \\ Dislocation of laser beam waists}

Another undesirable outcome of the optical aberration related to measurements of the tangential and radial velocity in the circular pipe is the dislocation of laser beam waists. This feature leads, on the one hand, to the wellknown fringe distortion in the measurement volume and, on the other, to the decrease of laser light intensity in the

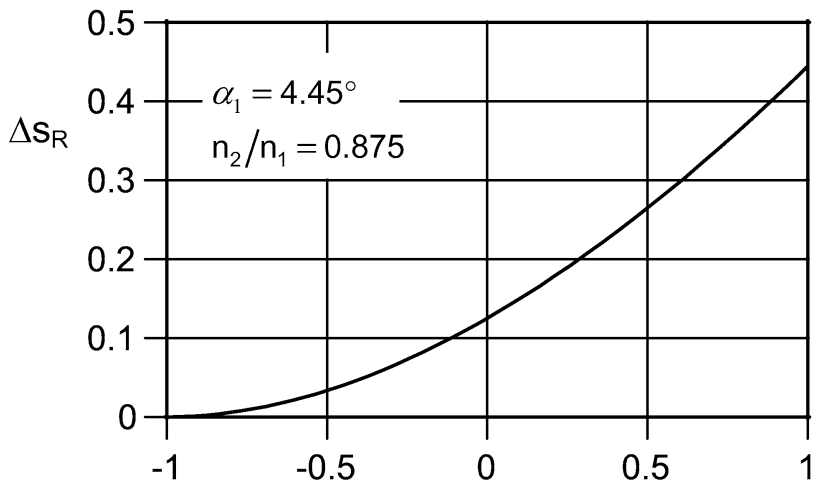

Position of the measurement volume $r / R$

Fig. 9. Relative differences in the position between the tangential and axial measurement volumes 
measurement volume and sequentially to a reduction in the signal quality. This type of signal disturbance contributes additionally to the deterioration of the signal quality owing to the optical aberration just treated above.

To quantify the beam waist dislocation the laser beams prior to the test fluid are assumed to be within design intent, i.e. any types of the optical aberration occurring at the cut-off plane on the outside of the circular pipe are negligible. Both cases of respective tangential and radial velocity measurements are separately considered.

\subsection{1}

\section{In measurements of tangential velocities $\boldsymbol{u}_{\boldsymbol{t}}$}

To calculate the laser beam waist, the laser beam is assumed first to suffer from the optical aberration through the refraction at the interface between the pipe wall and the fluid. This implies that because of astigmatism we need to distinguish between the meridional (tangential) and the sagittal focusing points of each laser beam $\left(p_{\mathrm{m}}\right.$ and $p_{\mathrm{s}}$ in Fig. 10). For this purpose, a new coordinate $\xi$ will be inserted, which begins at the beam intersection point $c$ and runs along the normal of the circular pipe, i.e. goes through the pipe axis. The laser beam considered is represented here by the unit vector $\vec{r}$. Its corresponding $\xi$ coordinates prior to and after the refraction are represented by $r_{1 \xi}=\cos \varepsilon_{1}$ and $r_{2 \xi}=\cos \varepsilon_{2}$, respectively (see Fig. 10).

Because the laser beam is thin, the intersection area on the cylindrical surface is assumed as the plane surface in order to simplify the beam refraction calculations. The respective tangential and sagittal focusing points of the refracted laser beam in the test fluid have their $\xi$ coordinates which, according to Zhang and Eisele (1996) for the plane interface, are given by:

$\xi_{m}=\xi_{o} \frac{n_{2}}{n_{1}} \frac{r_{2 \xi}^{3}}{r_{1 \xi}^{3}}$

and

$\xi_{s}=\xi_{o} \frac{n_{2}}{n_{1}} \frac{r_{2 \xi}}{r_{1 \xi}}$

Thereby $\xi_{o}$ denotes the $\xi$ coordinate of the virtual focusing point $m^{\prime}$, as given by

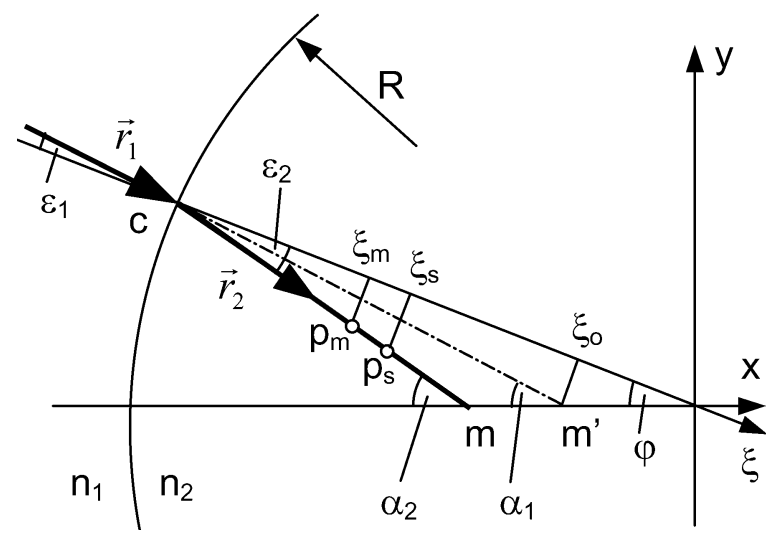

Fig. 10. Calculations of the laser beam waists and the measurement volume $\xi_{o}=c m^{\prime} \cdot \cos \varepsilon_{1}=\frac{R \cdot \sin \varphi}{\sin \alpha_{1}} r_{1 \xi}$

In the case of water flow in the circular pipe, it can be verified that $\left(r_{1 \xi}-r_{2 \xi}\right) / r_{1 \xi}<0.1 \%$ is nearly always satisfied, so that $r_{2 \xi} \approx r_{1 \xi}$ can be assumed. This points out that there is no need to differ from the tangential and sagittal focusing points. For the sake of obtaining the simple form of the results, however, Eq. 52 is applied to represent the $\xi$ coordinate of the unique laser beam waist:

$\xi_{w}=\xi_{s}=\xi_{o} \frac{n_{2}}{n_{1}} \frac{r_{2 \xi}}{r_{1 \xi}}$

On the refracted laser beam in the test fluid this beam waist lies at a distance from the intersection point c:

$\ell_{w}=\xi_{w} / \cos \varepsilon_{2}=\xi_{w} / r_{2 \xi}$

Because of the symmetry condition the measurement volume lies on the $x$ axis at $m$ and is at distance from the intersection point $\mathrm{c}$ :

$\ell_{M V}=\frac{R \cdot \sin \varphi}{\sin \alpha_{2}}$

The distance between the measurement volume and the waist of the laser beam is then given by $\ell_{M V}-\ell_{w}$ or in dimensionless form by

$\ell_{R}=\frac{\ell_{M V}-\ell_{w}}{R}=\frac{\sin \varphi}{\sin \alpha_{2}}-\frac{n_{2}}{n_{1}} \frac{\sin \varphi}{\sin \alpha_{1}}$

Clearly this distance depends on the local position of the measurement volume in the flow, as shown in Fig. 11, for example. Surprisingly, beyond the pipe centre there exits a large scaled dislocation of the beam waist. It implies low intensity of laser light and fringe distortion in the measurement volume. These two features clearly signify that the measurement of tangential velocities is more critical than that of axial velocities. In addition, it can be concluded from the values shown in Fig. 11in dimensionless form that the absolute beam waist dislocation $\left(\ell_{M V}-\ell_{w}\right)$ will be bigger, if pipes of large diameter are encountered.

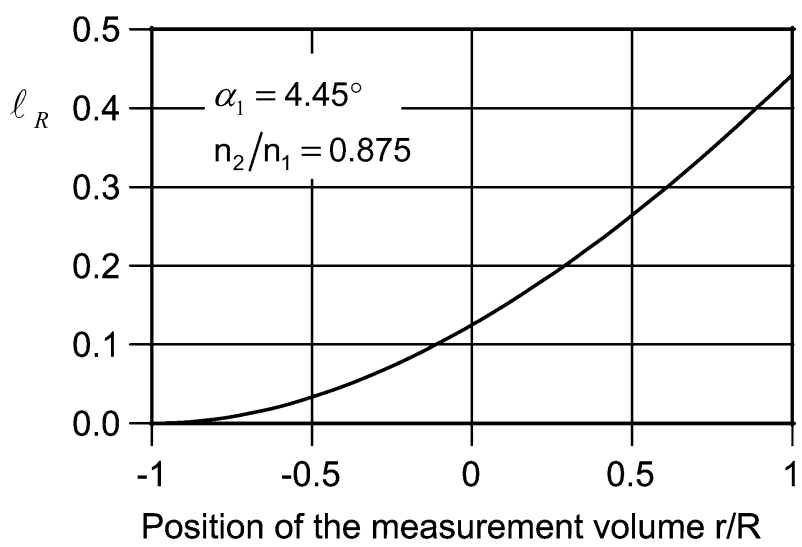

Fig. 11. Relative differences in the position between measurement volume and the laser beam waists for measurements of tangential velocities 
In comparing Fig. 11 with Fig. 9, it is evident that two curves are identical. In fact, by considering Eqs. 8, 9 and 10, Eq. $57 \mathrm{can}$ be reformed as:

$\ell_{R}=\frac{s_{2 x}}{R}-\frac{1}{\left(R / s_{2 x}-1\right)+n_{1} / n_{2}}$

which is exactly equal to Eq. 50 .

The fringe distortion in the measurement volume treated here exactly represents the first type of fringe distortions, at which the intersection of two laser beams takes place prior to or after their respective waists located at equal distance from the beam intersection point. In the current case of circular pipes, this occurrence is illustrated in Fig. 12. The fringe spacing in the measurement volume then linearly varies along the measurement volume. The corresponding errors involved in both the mean velocity and the flow turbulence measurements have already been exactly analysed by Zhang and Eisele (1998b), as given in the following equations for apparent mean and fluctuating velocities, respectively

$\frac{\bar{u}_{a}}{\bar{u}}=1+\frac{1}{3} \gamma^{2}$

$\frac{\sigma_{a}^{2}-\sigma^{2}}{\bar{u}^{2}}=\gamma^{2}\left(\frac{\sigma^{2}}{\bar{u}^{2}}+\frac{1}{3}\right)$

Thereby the fringe distortion number $\gamma$ is introduced, which represents the relative change in the fringe spacing at the end of the measurement volume to the initial fringe spacing and is usually below 0.02 . The analysis showed that the flow turbulence measurement could only be sensitively affected by the fringe distortion if the flow turbulence itself is low (say $T u<2 \%$ ), or if laminar flows are to be measured.

\subsection{2}

\section{In measurements of radial velocities $\boldsymbol{u}_{\boldsymbol{r}}$}

As in the above section a new coordinate $\xi$ is inserted, which begins at the beam intersection point $c$ and runs along the normal of the circular pipe (see Fig. 13). For simplicity only the sagittal focusing point of the refracted laser beam in the test fluid is considered as the beam waist $p_{\mathrm{w}}$. Similarly there is

$\xi_{\mathrm{W}}=\xi_{o} \frac{n_{2}}{n_{1}} \frac{r_{2 \xi}}{r_{1 \xi}}$

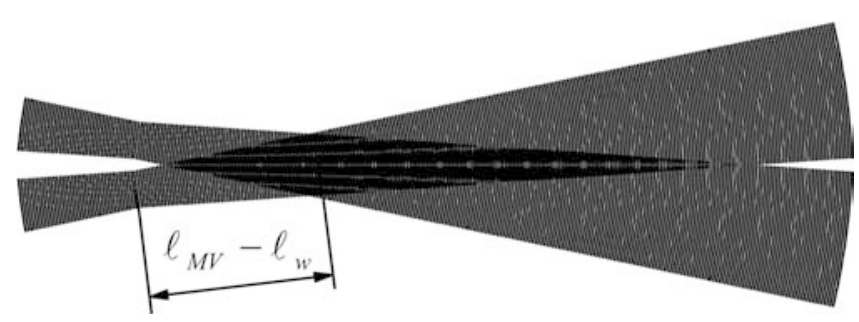

Fig. 12. Existence of the first type of the fringe distortion in the measurement volume for measurements of tangential velocities in the circular pipe

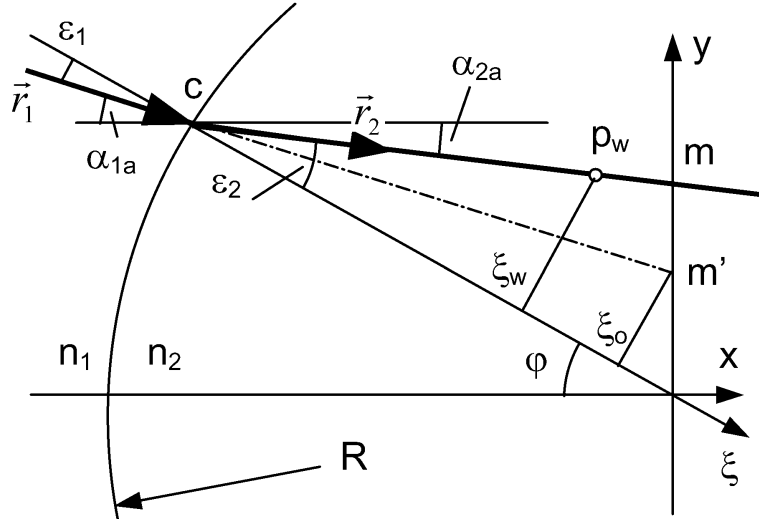

Fig. 13. Calculations of the laser beam waists $\left(p_{w}\right)$ and the measurement volume $(m)$

with

$\xi_{o}=\mathrm{cm}^{\prime} \cdot \cos \varepsilon_{1}=\frac{R \cdot \cos \varphi}{\cos \alpha_{1}} r_{1 \xi}$

On the refracted laser beam in the test fluid, the beam waist lies at $p_{\mathrm{w}}$ with a distance from the intersection point $\mathrm{c}$

$\ell_{\mathrm{w}}=\xi_{\mathrm{w}} / \cos \varepsilon_{2}=\xi_{\mathrm{w}} / r_{2} \xi$

The measurement volume $m$ is at a distance from the intersection point $\mathrm{c}$

$\ell_{M V}=\frac{R \cdot \cos \varphi}{\cos \alpha_{2 a}}$

The distance between the measurement volume $m$ and the laser beam waist $p_{w}$ is then given by $\ell_{M V}-\ell_{w}$. In dimensionless form and with subscripts $a$ and $b$ for laser beam $\mathrm{A}$ and $\mathrm{B}$, respectively, there are

$\ell_{R, a}=\frac{\cos \varphi_{a}}{\cos \alpha_{2 a}}-\frac{n_{2}}{n_{1}} \frac{\cos \varphi_{a}}{\cos \alpha_{1}}$
$\ell_{R, b}=\frac{\cos \varphi_{b}}{\cos \alpha_{2 b}}-\frac{n_{2}}{n_{1}} \frac{\cos \varphi_{b}}{\cos \alpha_{1 b}}$

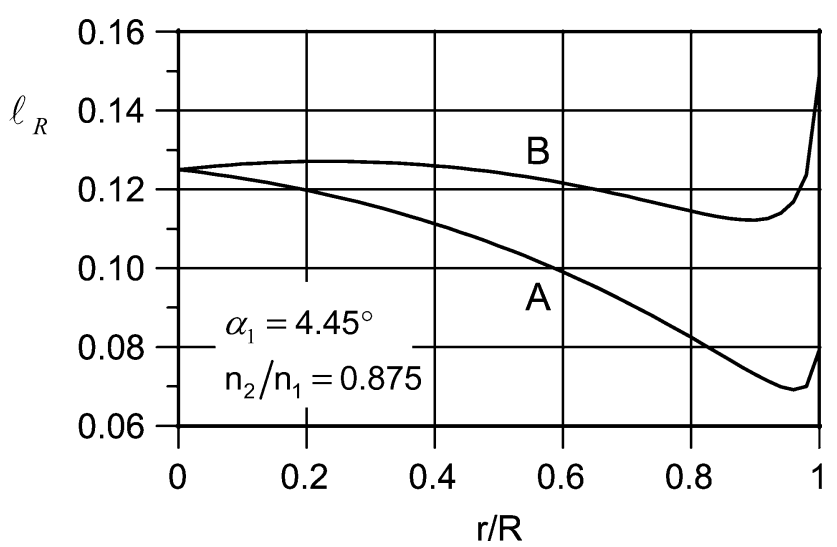

Fig. 14. Relative differences in the position between the measurement volume and the laser beam waists for measurements of radial velocities 
Clearly this distance depends on the local position $r / R$ of the measurement volume in the flow. Angles $\varphi_{a}, \varphi_{b}, \alpha_{2 a}$ and $\alpha_{2 b}$ and their dependences on $r / R$ are given by Eqs. 30, 31, 32, 33 and 34. Figure 14 shows the results calculated from Eqs. 65 and 66 for laser beam A and B, respectively. Because both laser beam waists are on the same side of the measurement volume and show no large differences in the distance to the measurement volume, it can be assumed that fringe distortion according to Fig. 12 occurs.

\section{6}

\section{Conclusions}

The optical performance of LDA technology in applications to circular pipes has been quantified with respect to the signal qualities and the operating guidelines. The entire quantifications of LDA performance are based on the use of the plane wall cut-off on the outside of the circular pipe, as such a plane wall cut-off has proved from experience to be very effective in enhancing the optical performance of LDA in this situation. Because of the optical aberration arising from the curved interface on the internal side of the circular pipe, measurements for axial, tangential and radial velocities must be carried out separately. For each velocity component, however, the measurements still suffer from optical aberrations, so that the velocity signals of sufficiently high quality could only be obtained within the depth of about two-thirds of the pipe diameter. A complete flow distribution along the pipe diameter could only be achieved by an additional measurement from the opposite side. This two-measurement feature straightforwardly represents the optical characteristic of the circular pipe. For measurements of axial velocities in the circular pipe no special care is taken, even if it occurs that the optical plane deviates from the pipe axis. For measurements of tangential and radial velocities, detailed operating guidelines have been presented with respect to the shift and the optical properties of the measurement volume. The large-scale dislocation of laser beam waists has been clarified to reveal the possible influences on both the signal quality and the measurement accuracy.

\section{References}

Boadway J, Karahan E (1981) Correction of laser Doppler anemometer readings for refraction at cylindrical interfaces. DISA Info. (26):4-6 Zhang Zh (1993) Theoretische Untersuchungen der Einsatzmöglichkeit für 3-dimensionale Laser-Doppler-Messungen in Kanalströmungen. Sulzer Innotec Bericht. Nr. STT.TB93.022. Winterthur, Schweiz

Zhang Zh (1995) Einfluss des Astigmatismus auf Laser Doppler Messungen. Sulzer Innotec Bericht. Nr. STT. TB95.022, Winterthur, Schweiz

Zhang Zh, Eisele K (1995) Off-axis alignment of an LDA-probe and the effect of astigmatism on the measurements. Exp Fluids 19:89-94

Zhang Zh, Eisele K (1996) The effect of astigmatism due to beam refractions in the formation of the measurement volume in LDA measurements. Exp Fluids 20:466-471

Zhang Zh, Eisele K (1998a) Further considerations of the astigmatism error associated with off-axis alignment of an LDA-probe. Exp Fluids 24:83-89

Zhang Zh, Eisele K (1998b) On the overestimation of the flow turbulence due to fringe distortion in LDA measurement volumes. Exp Fluids 25:371-374

Zhang Zh, Parkinson E (2002) LDA application and the dual-measurement-method in experimental investigations of the free surface jet at a model nozzle of a Pelton turbine. 10th. International Symposium on Applications of Laser Anemometry to Fluid Mechanics. Lisbon, Portugal 